\title{
Efficiency crisis of swift gamma-ray bursts with shallow X-ray afterglows: prior activity or time-dependent microphysics?
}

\author{
K. Ioka ${ }^{1}$, K. Toma ${ }^{1}$, R. Yamazaki ${ }^{2}$, and T. Nakamura ${ }^{1}$ \\ 1 Department of Physics, Kyoto University, Kyoto 606-8502, Japan \\ e-mail: ioka@tap.scphys.kyoto-u.ac.jp \\ 2 Department of Physics, Hiroshima University, Higashi-Hiroshima 739-8526, Japan
}

Received 31 January 2006 / Accepted 29 June 2006

ABSTRACT

\begin{abstract}
Context. Most X-ray afterglows of gamma-ray bursts (GRBs) observed by the Swift satellite have a shallow decay phase $\propto t^{-1 / 2}$ in the first few hours.

Aims. This is not predicted by the standard afterglow model and needs an explanation.

Methods. We discuss that the shallow decay requires an unreasonably high gamma-ray efficiency, $\gtrsim 75-90 \%$, within current models, which is difficult to produce by internal shocks. Such a crisis may be avoided if a weak relativistic explosion occurs $\sim 10^{3}-10^{6} \mathrm{~s}$ prior to the main burst or if the microphysical parameter of the electron energy increases during the shallow decay, $\epsilon_{\mathrm{e}} \propto t^{1 / 2}$. The former explanation predicts a very long precursor, while both prefer dim optical flashes from the reverse shock, as was recently reported. We also calculate the multi-wavelength afterglows and compare them with observations.

Results. No optical break at the end of the shallow X-ray decay indicates a preference for the time-dependent microphysics model with additionally decaying magnetic fields, $\epsilon_{B} \propto t^{-0.6}$.
\end{abstract}

Key words. gamma rays: bursts - gamma rays: theory - relativity - acceleration of particles - shock waves

\section{Introduction}

Recently the Swift satellite has allowed us to observe early afterglows of gamma-ray bursts (GRBs) in the first few hours after the burst (e.g., Tagliaferri et al. 2005; Burrows et al. 2005; Chincarini et al. 2005; Nousek et al. 2006; Cusumano et al. 2006; Hill et al. 2006; Vaughan et al. 2006; Barthelmy et al. 2005). This time window remains largely unexplored, and multiwavelength studies of early afterglows would reveal many questions concerning GRBs, such as the emission mechanism, nature of the central engine, and burst environment (Zhang et al. 2006; Nousek et al. 2006; Yamazaki et al. 2006; Toma et al. 2006; Kobayashi et al. 2005; Panaitescu et al. 2006a; Eichler \& Granot 2006; Granot \& Kumar 2006a; Lazzati \& Begelman 2005).

Early X-ray afterglows observed by the Swift X-Ray Telescope (XRT) have three kinds of canonical features that are not predicted by the standard model from the pre-Swift era (Nousek et al. 2006; Zhang et al. 2006). X-ray light curves show (i) a very steep initial decay ( $\propto t^{-\alpha_{1}}$ with $3 \lesssim \alpha_{1} \lesssim 5$ ) followed by (ii) a very shallow decay ( $\propto t^{-\alpha_{2}}$ with $0.2 \lessgtr \alpha_{2} \lesssim 0.8$ ) that connects to the conventional late afterglow, while about half of the afterglows have (iii) strong, rapid X-ray flares minutes to days after the burst (Piro et al. 2005; Burrows et al. 2005; Ioka et al. 2005; Fan \& Wei 2005).

The steep decay component is most likely the tail emission of the prompt GRBs and/or of the X-ray flares (Nousek et al. 2006; Zhang et al. 2006; Yamazaki et al. 2006). Even if the emitting surface stops shining, we continue to see photons coming from the region at large angles relative to our line-of-sight because the emitting surface has a curvature. Most photons from the large angles are not emitted in our direction because of relativistic beaming, so that the flux decays steeply. Since the emission region moves outward on the surface, the tail emission features, e.g., the decay index and smoothness, would diagnose the unknown GRB jet structure (Yamazaki et al. 2006).

The X-ray flares are considered to be produced by the long activity of the central engine up to the time of the flares (Burrows et al. 2005; Ioka et al. 2005). This is mainly because an afterglow cannot be variable with a large amplitude and a short timescale by itself, i.e., such as by the ambient density fluctuations and the inhomogeneous emitting surface, as concluded by the kinematic arguments (Ioka et al. 2005). However, the actual origin of the long activity is still under investigation (King et al. 2005; Perna et al. 2006).

The most enigmatic feature in early X-ray afterglows is the shallow decay of the light curve. So far two kinds of models are proposed for the shallow X-ray afterglows. One class of the models is the energy injection model (Nousek et al. 2006; Zhang et al. 2006; Granot \& Kumar 2006a), in which continuous energy is injected into the afterglow so that the flux decay becomes slower than the usual $\propto t^{-1}$. The injection may be caused by (a1) the long-lived central engine (Dai \& Lu 1998; Rees \& Mészáros 2000; Zhang \& Mészáros 2002) or (a2) the short-lived central engine ejecting shells with some ranges of Lorentz factors (Rees \& Mészáros 1998; Kumar \& Piran 2000; Sari \& Mészáros 2000; Zhang \& Mészáros 2002). The other class is (b) the inhomogeneous jet model (Toma et al. 2006; Eichler \& Granot 2006). In this model, early afterglows are not bright because the jet surface on the line-of-sight is dim and the surrounding off-axis region with ordinary brightness is observed later.

However, in all models, the shallow X-ray afterglows pose a serious problem, demanding an unreasonably high gamma-ray efficiency of the prompt GRBs (defined by $\epsilon_{\gamma} \equiv E_{\gamma} /\left(E_{\gamma}+E_{\mathrm{k}}\right)$, where $E_{\gamma}$ is the radiated prompt energy and $E_{\mathrm{k}}$ is the kinetic 
energy of the afterglow remaining after the burst), as explained in Sect. 2. Even before the Swift era, one considers that the gamma-ray efficiency of the prompt GRBs is relatively high, i.e., $\epsilon_{\gamma} \sim 50 \%$ or more (Lloyd-Ronning \& Zhang 2004), and develops internal shock models that can manage to produce such a high efficiency (Beloborodov 2000; Kobayashi \& Sari 2001). Since the required efficiency is further increased, we have a strong theoretical motivation to suspect the current models.

In this Letter we suggest two more kinds of possible models for shallow X-ray afterglows without invoking an unreasonably high gamma-ray efficiency. One is the prior activity model in Sect. 3.1, while the other is the time-dependent microphysics model in Sect. 3.2. The energetic afterglow model in Sect. 3.3 may also reduce the required efficiency. We also calculate the multi-wavelength afterglows in Sect. 4. We compare our results with observations and discuss implications in Sect. 5.

\section{Efficiency crisis}

Let us show that a high gamma-ray efficiency is necessary to explain the shallow X-ray afterglows within models proposed so far. Here we should note that the flux decay is shallower than $\propto t^{-1}$ and therefore more time-integrated energy is radiated at a later time. In this section we assume that electrons are accelerated to a power-law distribution $N\left(\gamma_{\mathrm{e}}\right) \propto \gamma_{\mathrm{e}}^{-2}$ and X-rays arise from fast cooling electrons, so that the X-ray luminosity is proportional to the bolometric one. Note that the lack of spectral evolution across the X-ray break indicates that these breaks are not produced by the passage of a spectral break.

(a1) First we consider the energy injection model caused by the long-lived central engine. If there is no injection, the light curve decays as $\propto t^{-1}$ after the peak time $t_{\mathrm{dec}} \sim \max \left[T, t_{\gamma}\right]$, where $T$ is the burst duration,

$t_{\gamma}=\left(\frac{3 E_{\mathrm{k}}}{256 \pi \gamma^{8} n m_{\mathrm{p}} c^{5}}\right)^{1 / 3} \sim 100 E_{\mathrm{k}, 53}^{1 / 3} \gamma_{2}^{-8 / 3} n^{-1 / 3} \mathrm{~s}$

is the time to collect $\gamma^{-1}$ of the ejecta mass, $\gamma_{2}=10^{2} \gamma$ is the Lorentz factor of the ejecta, $n$ is the ambient density, and $E_{\mathrm{k}}=$ $10^{53} E_{\mathrm{k}, 53}$ erg is the afterglow energy (Sari 1997). The peak time $\left(t_{\mathrm{dec}} \sim 1-10^{2} \mathrm{~s}\right)$ is typically before the end of the shallow decay $\left(t_{\mathrm{s}} \sim 10^{3}-10^{4} \mathrm{~s}\right)$.

If the engine continues to eject outflows after the prompt burst, the outflows add energy to the external shock. Then the afterglow decay becomes shallower $\left(\propto t^{-1 / 2}\right)$ than that for no injection $\left(\propto t^{-1}\right)$. Since the decay $\propto t^{-1 / 2}$ is shallower than $\propto t^{-1}$, the time-integrated injected energy $E_{\text {inj }}$ is larger than the initial afterglow energy $E_{\mathrm{k}}$ by a factor of $E_{\mathrm{inj}} / E_{\mathrm{k}} \sim\left(t_{\mathrm{s}} / t_{\mathrm{dec}}\right)^{1 / 2} \sim 3-10$. Since the burst energy is comparable to the afterglow energy after injection $E_{\gamma} \sim E_{\mathrm{inj}} \sim 3-10 E_{\mathrm{k}}$, the gamma-ray efficiency is corrected upward as $\epsilon_{\gamma}=E_{\gamma} /\left(E_{\gamma}+E_{\mathrm{k}}\right) \gtrsim 75-90 \%$. Such a high efficiency is difficult to explain with realistic internal shock models (Kobayashi \& Sari 2001).

(a2) Next we consider the energy injection model caused by a short-lived central engine with some ranges of Lorentz factors of ejected shells. After the internal shocks, shells are rearranged so that outer shells are faster and inner shells are slower. This configuration may also occur if the central engine ejects faster shells earlier. Outer shells are slowed down by making the external shock. Once the Lorentz factor of the shocked shell drops below that of the slower shell, the slower shell catches up with the shocked shell, injecting energy into the forward shock. Thus the injection time $t_{\mathrm{i}}$ of a shell with a Lorentz factor $\gamma$ is about $t_{\mathrm{i}} \sim t_{\gamma}$ in Eq. (1), replacing $E_{\mathrm{k}}$ with the time-integrated injected energy.
The shallow phase continues until the energy in slower shells becomes less than the time-integrated injected energy. Then, by equating the final time of the shallow phase $t_{\mathrm{s}} \sim 10^{3}-10^{4} \mathrm{~s}$ with the injection time $t_{\mathrm{i}} \sim t_{\gamma}$ in Eq. (1), we can estimate the Lorentz factor $\gamma_{\text {peak }}$ in which most energy resides as $\gamma_{\text {peak }} \sim 30-50$. Since $t_{\mathrm{i}} \sim t_{\gamma} \propto \gamma^{-8 / 3}$ and the time-integrated energy grows as $\sim t^{1 / 2}$, the energy distribution is given by $\mathrm{d} E / \mathrm{d} \ln \gamma \propto t^{1 / 2} \propto$ $\gamma^{-4 / 3}$ for $\gamma>\gamma_{\text {peak }}$ (Granot \& Kumar 2006a). Therefore, the energy in the shells with $\gamma \gtrsim 100$ is smaller than the total injected energy $E_{\text {inj }}$ by a factor of 3-10. Now we recall that only shells with $\gamma \gtrsim 100$ can make the prompt burst because of the compactness problem (Lithwick \& Sari 2001). Then the afterglow energy remaining after the burst $E_{\mathrm{k}}$ is a factor 3-10 smaller than the total injected energy, i.e., $E_{\mathrm{inj}} \sim 3-10 E_{\mathrm{k}}$, while the burst energy is comparable to the afterglow energy after injection $E_{\gamma} \sim E_{\text {inj. }}$. Again we find that the corrected gamma-ray efficiency is very high, $\epsilon_{\gamma}=E_{\gamma} /\left(E_{\gamma}+E_{\mathrm{k}}\right) \gtrsim 75-90 \%$.

One may think that the gamma-ray efficiency is not so high if the initial energy distribution $\mathrm{d} E / \mathrm{d} \ln \gamma$ peaks at $\gamma_{\text {peak }}>100$ and the peak moves to $\gamma_{\text {peak }} \sim 30$ after internal shocks. However, in order for the peak Lorentz factor $\gamma_{\text {peak }}$ to move down to $\gamma_{\text {peak }} \sim 30$, the shells of $\gamma>100$ have to interact with shells of $\gamma \sim 30$. Since the internal shock radius is determined by the lower Lorentz factor, the internal shocks occur deeply in the optically thick region, and therefore we cannot avoid the compactness problem.

(b) Toma et al. (2006) have completely discussed the inhomogeneous jet model. This model also needs a high gamma-ray efficiency $\epsilon_{\gamma}=E_{\gamma} /\left(E_{\gamma}+E_{\mathrm{k}}\right) \gtrsim 75 \%$. Therefore, all current models face an efficiency crisis!

\section{Possible solutions}

\subsection{Prior activity model}

Since the afterglow energy after the shallow phase is more or less similar to the burst energy, we are tempted into considering that both types of energy have the same origin. Then, to suppress the flux of the early afterglow, we logically have two choices: (A) the kinetic energy of the ejecta is not converted into the internal energy so much in the early phase or (B) even if the kinetic energy is converted into the internal energy it is not radiated away so much. The choice (B) will be discussed in Sect. 3.2.

For the choice (A) one may easily think of reducing the ambient density $n$ because the kinetic energy is released when the ejecta is decelerated and the deceleration time is given by $t_{\mathrm{dec}} \sim$ $t_{\gamma} \propto n^{-1 / 3}$ in Eq. (1). However in this case, the early afterglow does not show a decaying feature but a rising one (Sari 1997). If we adjust the ambient density so as to have a shallow decay, we need an unrealistic density profile that drops outward and does not connect to the conventional density for the late afterglow. Therefore, we cannot simply reduce the ambient density. However, how about changing both the density and velocity of the ambient matter? Such a situation is not implausible if a prior explosion occurs before the observed prompt GRBs, for example. It is not unreasonable to consider such a prior activity because the X-ray flares suggest that the engine activity lasts very long after the burst (Burrows et al. 2005; Ioka et al. 2005) and it may be also present before the burst. Actually, a sizable fraction of GRBs may have precursor activities (Lazzati 2005). A prior activity may be also expected in the supranova model (Vietri \& Stella 1998), although the ambient matter is not relativistic in this model. In the collapsar model such a prior activity might 


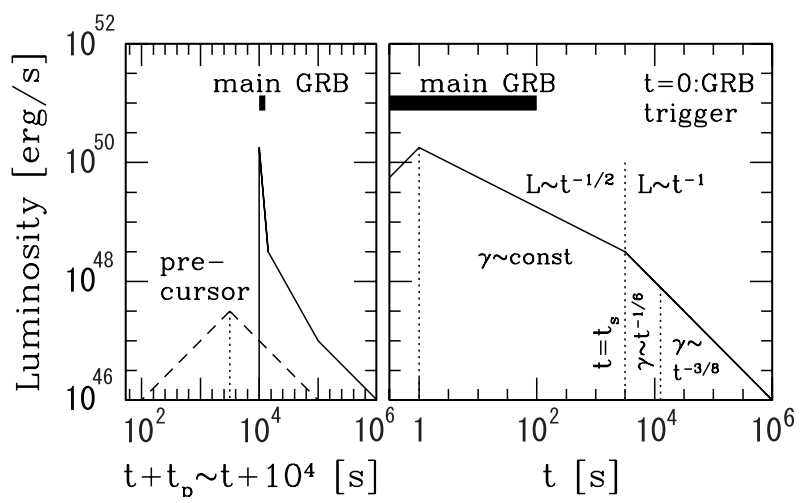

Fig. 1. The afterglow luminosity as a function of time before the main burst (left) and after the main burst (right) in the prior activity model. We set $t=0$ at the beginning of the main burst (thick line). A precursor (dashed line) is produced by the forward shock due to the prior ejecta launched at $t=-t_{\mathrm{p}} \sim-10^{4} \mathrm{~s}$. The forward shock emission due to the main burst ejecta (solid line) has a shallow decay $\propto t^{-1 / 2}$ before the burst ejecta is decelerated by the prior ejecta $t<t_{\mathrm{s}}$ in Eq. (11), and after that it has the conventional decay $\propto t^{-1}$.

arise if the main burst is produced by the fallback of the ejected matter.

To demonstrate the plausibility of the prior activity model, we consider the following simple model. We assume that an explosion occurs at $t=-t_{\mathrm{p}} \sim-10^{4} \mathrm{~s}$ (where we set $t=0$ as the burst trigger) and that mass

$M\left(<\gamma_{\mathrm{p}}\right) \propto \gamma_{\mathrm{p}}^{\alpha}$

with Lorentz factors less than $\gamma_{\mathrm{p}}$ is ejected, where we assume $\alpha>0$ and $\gamma_{\mathrm{p}}<\gamma_{\max } \sim 30$. The energy associated with that mass is $E\left(<\gamma_{\mathrm{p}}\right)=\gamma_{\mathrm{p}} M c^{2} \propto \gamma_{\mathrm{p}}^{\alpha+1}$. Since $\alpha>0$, almost all energy is concentrated near $\gamma_{\max }$. We also assume that a prior explosion is weaker than the main burst, $E\left(<\gamma_{\max }\right) \equiv E_{\mathrm{p}} \sim 10^{52} \mathrm{erg}<$ $E_{\gamma} \sim 10^{53} \mathrm{erg}$. The ejected mass sweeps the ambient density making an external shock. The deceleration begins at $t \sim-t_{\mathrm{p}}+$ $10^{4} E_{\mathrm{p}, 52}^{1 / 3} \gamma_{\max , 1.5}^{-8 / 3} n^{-1 / 3} \mathrm{~s}$ from Eq. (1), and then the Lorentz factor and radius of the external shock evolve as

$$
\begin{aligned}
\gamma_{\mathrm{p}} & \sim 30 E_{\mathrm{p}, 52}^{1 / 8} n^{-1 / 8}\left[\left(t+t_{\mathrm{p}}\right) / 10^{4} \mathrm{~s}\right]^{-3 / 8}, \\
R & \sim 10^{17} E_{\mathrm{p}, 52}^{1 / 4} n^{-1 / 4}\left[\left(t+t_{\mathrm{p}}\right) / 10^{4} \mathrm{~s}\right]^{1 / 4} \mathrm{~cm} .
\end{aligned}
$$

Since the explosion is weak, its afterglow is not so bright (see Fig. 1 and Sect. 5). Note that the Blandford \& McKee (1976) solution has the mass profile $M\left(<\gamma_{\mathrm{p}}\right) \propto \gamma_{\mathrm{p}}^{3 / 2}$ near the shock front and the index $\alpha$ is larger far from the shock.

We assume that the ejecta of the prompt burst at $t=0$ is faster than the prior ejecta, i.e., $\gamma>\gamma_{\max }$. Before catching up with the external shock, the burst ejecta will collide with the slower ejecta at a radius $R_{\mathrm{p}} \sim c t_{\mathrm{p}} \gamma_{\mathrm{p}}^{2}$. The relative Lorentz factor between the burst and slower ejecta is about $\eta \sim \gamma / \gamma_{\mathrm{p}}$ for $\gamma \gg$ $\gamma_{\mathrm{p}} \gg 1$, while the ratio of the comoving density is given by

$f \equiv \frac{n_{\mathrm{k}}}{n_{\mathrm{p}}} \sim \frac{E_{\mathrm{k}}}{E\left(<\gamma_{\mathrm{p}}\right)} \propto \gamma_{\mathrm{p}}^{-\alpha-1}$,

where $n_{\mathrm{k}}\left(n_{\mathrm{p}}\right)$ is the density of the burst (slower) ejecta and where we assume the slower ejecta is cold before the collision since it is not shocked.

Let us assume $\eta^{2}<f$ in the early phase. Then the reverse shock is Newtonian (Sari \& Piran 1995). The burst ejecta is not decelerated and keeps its Lorentz factor $\gamma \sim$ const. The internal energy is mainly released in the forward shock, which is given by

$E_{\mathrm{i}} \sim M\left(<\gamma_{\mathrm{p}}\right) \frac{\gamma^{2}}{\gamma_{\mathrm{p}}} \propto \gamma_{\mathrm{p}}^{\alpha-1}$.

The radiation from the collision is observed at

$t \sim \frac{R_{\mathrm{p}}}{\gamma^{2}} \sim t_{\mathrm{p}} \frac{\gamma_{\mathrm{p}}^{2}}{\gamma^{2}} \sim 10^{3} t_{\mathrm{p}, 4} \gamma_{2}^{-2} \gamma_{\mathrm{p}, 1.5}^{2} \mathrm{~s}$,

where $t_{\mathrm{p}}=10^{4} t_{\mathrm{p}, 4} \mathrm{~s}$ and $\gamma_{\mathrm{p}}=10^{1.5} \gamma_{\mathrm{p}, 1.5} \sim 30 \gamma_{\mathrm{p}, 1.5}$, and hence $\gamma_{\mathrm{p}} \propto t^{1 / 2}$. From Eqs. (6) and (7), the bolometric kinetic luminosity is given by

$L \sim \frac{E_{\mathrm{i}}}{t} \propto \frac{\gamma_{\mathrm{p}}^{\alpha-1}}{t} \propto t^{(\alpha-3) / 2}$

Therefore, assuming that the X-ray luminosity is proportional to the bolometric kinetic one, we can explain the shallow decay if $\alpha \sim 1.5-2.5$ (see Fig. 1). If the X-ray luminosity is not proportional to the bolometric kinetic one, the light curve is given by Eq. (22) in Sect. 4.

For an index $\alpha>1$, the ratio $f / \eta^{2} \propto \gamma_{\mathrm{p}}^{1-\alpha}$ is a decreasing function of $\gamma_{\mathrm{p}}$. The ratio $f / \eta^{2}$ becomes less than unity as the shock expands if $\min \left(f / \eta^{2}\right) \sim \min \left[E_{\mathrm{k}} \gamma_{\mathrm{p}}^{2} / E\left(<\gamma_{\mathrm{p}}\right) \gamma^{2}\right] \sim$ $E_{\mathrm{k}} \gamma_{\max }^{2} / E_{\mathrm{p}} \gamma^{2}<1$ is satisfied. Therefore, the initially Newtonian reverse shock becomes relativistic. At this point the reverse shock also crosses the burst ejecta because the crossing radius is given by $R_{\Delta} \sim\left(f / \eta^{2}\right)^{1 / 2} R$ (Sari \& Piran 1995). Beyond the crossing radius, we can use a simple two mass model to estimate the Lorentz factor of the forward shock due to the burst ejecta,

$\gamma \sim \gamma_{\mathrm{p}}\left(\frac{E_{\mathrm{k}}}{E\left(<\gamma_{\mathrm{p}}\right)}\right)^{1 / 2} \propto \gamma_{\mathrm{p}}^{(1-\alpha) / 2} \propto t^{(1-\alpha) / 2(1+\alpha)}$.

Since $\alpha>1$, the forward shock due to the burst ejecta is decelerating. After the deceleration, the internal energy released in the forward shock is comparable to the energy of the burst ejecta $\sim E_{\mathrm{k}}$. Then the bolometric kinetic luminosity evolves as

$L \sim E_{\mathrm{k}} / t \propto t^{-1}$,

which is the conventional decay after the shallow phase. The time when the conventional decay begins is estimated from Eq. (7) with $\gamma_{\mathrm{p}}$ satisfying $f / \eta^{2} \sim 1$, i.e.,

$t_{\mathrm{s}} \sim t_{\mathrm{p}} \frac{E\left(<\gamma_{\mathrm{p}}\right)}{E_{\mathrm{k}}} \lesssim 10^{3} t_{\mathrm{p}, 4} E_{\mathrm{p}, 52} E_{\mathrm{k}, 53}^{-1} \mathrm{~s}$.

This is about $\sim 10^{3} \mathrm{~s}$ for our parameters and reproduces the observations (see Fig. 1).

Finally, the forward shock due to the burst ejecta overtakes the outermost forward shock due to the prior ejecta. At this radius the Lorentz factor $\gamma$ of the forward shock due to the burst ejecta in Eq. (9) is the same as that in the absence of the prior explosion. Therefore we have the same luminosity evolution in Eq. (10) after the forward shock drives into the ambient medium. (For more strict arguments, calculations like those in Zhang \& Mészáros 2002, are necessary.) Since the prior explosion has less energy than the prompt burst, the final afterglow energy is comparable to the initial afterglow energy $E_{\mathrm{k}}$, and hence we have no efficiency crisis.

In summary, a shallow light curve can be reproduced without the efficiency crisis if a small explosion with less energy than the main burst occurs $t_{\mathrm{p}} \sim 10^{3}-10^{6} \mathrm{~s}$ before the burst. The shallow 
phase ends at around $t_{\mathrm{s}} \sim 10^{3}-10^{4} \mathrm{~s}$ in Eq. (11), and this time marks the beginning of the deceleration of the burst ejecta due to the prior ejecta. The decay index of the shallow phase in Eq. (8) is mainly determined by the mass distribution of the prior ejecta in Eq. (2). More detail calculations are given in Sect. 4 and are compared with observations in Sect. 5.

\subsection{Time-dependent microphysics model}

The other possibility to obtain the shallow X-ray afterglow without the efficiency crisis is to vary the microphysical parameters, such as the energy fraction that goes into electrons $\epsilon_{\mathrm{e}}$ and magnetic fields $\epsilon_{B}$, during the observations. Even if the burst ejecta is decelerated and the internal energy is released, most internal energy is initially carried by protons. Without transferring the proton energy into electrons and magnetic fields, little radiation is emitted since protons are inefficient emitters.

So far we usually assume that the microphysical parameters are not varying and in fact, constant $\epsilon_{\mathrm{e}}$ and $\epsilon_{B}$ are consistent with the observations of late afterglows (Yost et al. 2003). However, since the first few hours after the burst is an unexplored period, we should check the constancy of microphysics observationally in this time interval without having any prejudice. Also, on the theoretical side, the mechanism of the energy transfer from protons to electrons and magnetic fields in the relativistic shocks is not well understood from the first principles. Although recent particle simulations have demonstrated that the magnetic fields are generated by the Weibel instability in collisionless shocks (Medvedev \& Loeb 1999; Silva et al. 2003; Kato 2005), the long term evolution up to the time of the actual observation is beyond the current computer power. In addition, simulations have not succeeded in reproducing $\epsilon_{\mathrm{e}} \sim 0.1$ (but see Hededal 2005), probably because the grid size is not small enough for resolving the radiation wavelength, and hence the coherent effects are not properly calculated (Ioka 2005). Since the coherent effects could depend on the Lorentz factor of the shock (Ioka 2005), the electron energy fraction may vary in the early afterglow.

If the index of the power-law electron distribution $p$ is about $p \sim 2$ as usual and fast cooling electrons emit X-rays, the X-ray luminosity $L_{X}$ is given by the bolometric kinetic luminosity $L$ as

$L_{\mathrm{X}} \sim \epsilon_{\mathrm{e}} L$,

and does not depend on the magnetic energy fraction $\epsilon_{B}$ so much $\left(L_{\mathrm{X}} \propto \epsilon_{B}^{(p-2) / 4}\right)$. Since $L \propto t^{-1}$, the shallow $\mathrm{X}$-ray light curve $L_{\mathrm{X}} \propto t^{-1 / 2}$ suggests that the electron energy fraction evolves as

$\epsilon_{\mathrm{e}} \propto t^{1 / 2}$

which is saturated at the equipartition value $\epsilon_{\mathrm{e}} \sim 0.1-1$ when the shallow phase ends. Note that the initial value of $\epsilon_{\mathrm{e}}$ at $t \sim$ $1-100 \mathrm{~s}$ is still larger than the minimum energy fraction $\epsilon_{\mathrm{e}, \min }=$ $m_{\mathrm{e}} / m_{\mathrm{p}} \sim 10^{-3}$. More detail calculations are presented in Sect. 4.

\subsection{Energetic afterglow model}

The gamma-ray efficiency $\epsilon_{\gamma}=E_{\gamma} /\left(E_{\gamma}+E_{\mathrm{k}}\right)$ can be reduced if the kinetic energy of the afterglows $E_{\mathrm{k}}$ is actually much larger than previously thought (Fan \& Piran 2006; Granot et al. 2006b). This could be caused by the following possibilities, (i) the standard afterglow model has some ambiguities. In particular, LloydRonning \& Zhang (2004) take a relatively large typical synchrotron frequency $\nu_{\mathrm{m}}$, which increases $\epsilon_{\gamma}$; (ii) almost all energy could be radiated by the inverse Compton emission that is not observed; (iii) only a part of the electrons $\zeta_{\mathrm{e}}$ could be accelerated
(Papathanassiou \& Mészáros 1996). Thus, the afterglow fitting could actually give a larger kinetic energy (Eichler \& Waxman 2005).

Regardless, in this model the kinetic energy of the afterglow should be $\sim 10$ times larger than previously thought for a moderate gamma-ray efficiency $\epsilon_{\gamma} \sim 0.1$. In other words, the total energy of the gamma-ray bursts is $\gtrsim 10^{52} \mathrm{erg}$ rather than $\sim 10^{51} \mathrm{erg}$. Note that this model itself does not explain the shallow decay of the X-ray afterglow. We also have to consider time-dependent microphysical parameters $\left(\epsilon_{\mathrm{e}}, \epsilon_{B}, \zeta_{\mathrm{e}}\right)$, the energy injection, or the inhomogeneous jet.

\section{Multi-wavelength afterglows}

First, let us consider the prior activity model. There are three phases in this model. (i) The first one is the $\gamma \sim$ const. phase, which corresponds to the shallow X-ray phase; (ii) the second one follows after the reverse shock crosses the burst ejecta; (iii) finally, the conventional evolution in the constant density medium begins after the burst ejecta overtakes the outermost forward shock due to the prior ejecta. In the first phase (i), we estimate the comoving density of the pre-shocked matter $n_{\mathrm{p}}$, the typical Lorentz factor of shocked electrons $\gamma_{\mathrm{m}}$, the comoving magnetic field in the shocked region $B$, and the total number of emitting electrons $N_{\mathrm{e}}$ as

$$
\begin{aligned}
n_{\mathrm{p}} & =\frac{M\left(<\gamma_{\mathrm{p}}\right)}{4 \pi m_{\mathrm{p}} R_{\mathrm{p}}^{3} / \gamma_{\mathrm{p}}} \propto \gamma_{\mathrm{p}}^{\alpha-5} \propto t^{(\alpha-5) / 2}, \\
B & =\left(32 \pi m_{\mathrm{p}} \epsilon_{B} n_{\mathrm{p}}\right)^{1 / 2} \eta c \propto \epsilon_{B}^{1 / 2} t^{(\alpha-7) / 4}, \\
\gamma_{\mathrm{m}} & =\epsilon_{\mathrm{e}} \frac{p-2}{p-1} \frac{m_{\mathrm{p}}}{m_{\mathrm{e}}} \eta \propto \epsilon_{\mathrm{e}} \gamma_{\mathrm{p}}^{-1} \propto \epsilon_{\mathrm{e}} t^{-1 / 2}, \\
\gamma_{\mathrm{c}} & =\frac{6 \pi m_{\mathrm{e}} c}{\sigma_{T} \gamma B^{2} t} \propto \epsilon_{B}^{-1} t^{(5-\alpha) / 2}, \\
N_{\mathrm{e}} & =\frac{M\left(<\gamma_{\mathrm{p}}\right)}{m_{\mathrm{p}}} \propto \gamma_{\mathrm{p}}^{\alpha} \propto t^{\alpha / 2} .
\end{aligned}
$$

Then, according to the standard afterglow theory (Sari et al. 1998), we can calculate the spectral evolutions as

$$
\begin{aligned}
v_{\mathrm{m}} & =\frac{\gamma \gamma_{\mathrm{m}}^{2} q B}{2 \pi m_{\mathrm{e}} c} \sim 1 \times 10^{12} \mathrm{~Hz} \epsilon_{\mathrm{e},-1}^{2} \epsilon_{B,-2}^{1 / 2} t_{3}^{(\alpha-11) / 4}, \\
v_{\mathrm{c}} & =\frac{\gamma \gamma_{\mathrm{c}}^{2} q B}{2 \pi m_{\mathrm{e}} c} \sim 8 \times 10^{15} \mathrm{~Hz} \epsilon_{B,-2}^{-3 / 2} t_{3}^{(13-3 \alpha) / 4}, \\
F_{\gamma, \max } & =\frac{N_{\mathrm{e}} m_{\mathrm{e}} c^{2} \sigma_{T} \gamma B}{12 \pi D^{2} q} \sim 0.4 \mathrm{Jy} \epsilon_{B,-2}^{1 / 2} t_{3}^{(3 \alpha-7) / 4},
\end{aligned}
$$

where $\epsilon_{\mathrm{e},-1}=\epsilon_{\mathrm{e}} / 0.1, \epsilon_{B,-2}=\epsilon_{B} / 0.01, t_{3}=t / 10^{3} \mathrm{~s}$, and we adopt $p=2.2, \gamma=100, \gamma_{\max }=30, E_{\mathrm{p}}=10^{52} \mathrm{erg}, t_{\mathrm{p}}=10^{4} \mathrm{~s}$, and $\alpha=2$. Therefore, the X-ray light curve is given by

$$
\begin{aligned}
F_{v_{\mathrm{c}}<v}= & F_{v, \max }\left(v_{\mathrm{c}} / v_{\mathrm{m}}\right)^{(1-p) / 2}\left(v / v_{\mathrm{c}}\right)^{-p / 2} \\
& \propto t^{\frac{\alpha-3}{2}+\frac{(\alpha-11)(p-2)}{8}},
\end{aligned}
$$

which is consistent with Eq. (8) since $F_{v_{c}<\nu} \propto t^{(\alpha-3) / 2}$ for $p=2$. (Note that the luminosity per logarithmic frequency is constant for $p=2$ and so the X-ray luminosity is proportional to the bolometric one.) The optical light curve is

$$
F_{v_{\mathrm{m}}<v<v_{\mathrm{c}}}=F_{v, \max }\left(v / v_{\mathrm{m}}\right)^{(1-p) / 2} \propto t^{\frac{7 \alpha-25}{8}+\frac{(\alpha-11)(p-2)}{8}} .
$$

After the reverse shock crosses the burst ejecta in the prior activity model (i.e., in phase (ii)), the Lorentz factor of the forward 
shock evolves according to Eq. (9), and so we have $\gamma_{\mathrm{p}} \propto t^{1 /(1+\alpha)}$ with Eq. (7). By using the same Eqs. (14)-(21) with different $t$ dependences of $\gamma$ and $\gamma_{\mathrm{p}}$, we can find

$$
\begin{gathered}
v_{\mathrm{m}} \propto t^{(-3 \alpha-7) / 2(1+\alpha)}, \\
v_{\mathrm{c}} \propto t^{(-3 \alpha+13) / 2(1+\alpha)}, \\
F_{v, \max } \propto t^{(\alpha-5) / 2(1+\alpha)} .
\end{gathered}
$$

Therefore, the X-ray light curve is

$F_{v_{\mathrm{c}}<v} \propto t^{-1-\frac{(3 \alpha+7)(p-2)}{4(1+\alpha)}}$,

which is consistent with Eq. (10), since $F_{v_{\mathrm{c}}<v} \propto t^{-1}$ for $p=2$. The optical light curve is

$$
F_{v_{\mathrm{m}}<v<v_{\mathrm{c}}} \propto t^{-\frac{\alpha+17}{4(1+\alpha)}-\frac{(3 \alpha+7)(p-2)}{4(1+\alpha)}} .
$$

After the burst ejecta runs into the interstellar medium (i.e., in phase (iii)), we note the conventional light curve as

$F_{v_{\mathrm{c}}<v} \propto t^{-1-3(p-2) / 4}, \quad F_{v_{\mathrm{m}}<v<v_{\mathrm{c}}} \propto t^{-3(p-1) / 4}$.

The reverse shock emission in the prior activity model peaks when the shock crosses the burst ejecta in Eq. (11). Since the reverse shock is relativistic at $t \sim t_{\mathrm{s}}$, the energy and energy density are similar to that of the forward shock. The thermal Lorentz factor is $\eta^{-1}$ times smaller, while the electron number is $\eta$ times larger than that of the forward shock. Then $v_{\mathrm{m}}, v_{\mathrm{c}}$, and $F_{v \text {, max }}$ are $\eta^{-2}, 1$, and $\eta$ times larger than that of the forward shock, respectively. Therefore, the reverse shock emission is dominated by the forward shock emission, at least in the optical band.

Second, let us consider the time-dependent microphysics model. For general arguments, we parameterize

$\epsilon_{\mathrm{e}} \propto t^{\beta_{\mathrm{e}}}, \quad \epsilon_{B} \propto t^{\beta_{B}}$,

in the shallow X-ray phase. Since the X-ray band is typically above $v_{\mathrm{c}}$ and the optical band is between $v_{\mathrm{m}}$ and $v_{\mathrm{c}}$, the X-ray and optical light curves are given by

$$
\begin{aligned}
F_{v_{\mathrm{c}}<v} & \propto t^{-1+\beta_{\mathrm{e}}+\frac{\left(4 \beta_{\mathrm{e}}+\beta_{B}-3\right)(p-2)}{4}}, \\
F_{v_{\mathrm{m}}<v<v_{\mathrm{c}}} & \propto t^{\frac{4 \beta_{\mathrm{e}+3 \beta_{B}-3}}{4}+\frac{\left(4 \beta_{\mathrm{e}}+\beta_{B}-3\right)(p-2)}{4}},
\end{aligned}
$$

respectively. After the shallow X-ray phase, the optical light curve returns to the conventional decay in Eq. (29). The reverse shock emission is dominated by the forward shock emission in the optical band, since $v_{\mathrm{m}}, v_{\mathrm{c}}$, and $F_{v, \text { max }}$ are $\gamma^{-2}, 1$, and $\gamma$ times larger than that of the forward shock, respectively, if the microphysical parameters evolve in a similar manner to the forward shock. We do not consider the energetic afterglow model in this Letter since the model itself does not explain the shallow X-ray decay.

\section{Discussion}

We have discussed that the shallow decay of the X-ray afterglow requires an unreasonably high gamma-ray efficiency, $\gtrsim 75-90 \%$, within current models (the energy injection model and the inhomogeneous jet model). Since such an efficiency is difficultly produced by internal shocks, we have suggested other possibilities, i.e., the prior activity model and the time-dependent microphysics model, that have no efficiency crises. We have also calculated the multi-wavelength afterglows in these models.

Let us compare the multi-wavelength afterglows with observations, although the multi-wavelength observations of the early afterglows are not yet very abundant. For several events, the X-ray and optical decay indices are around - $(0.5-1)$ and -(0.4-0.8), respectively, in the shallow X-ray phase (Panaitescu et al. 2006b). In the prior activity model, such behavior occurs if $p \sim 3$ (the power-law index of the electron distribution) and $\alpha \sim 3.8$ (in Eq. (2)), which give indices of $\sim-0.5$ (X-ray) and $\sim-0.7$ (optical) from Eqs. (22) and (23). However, the spectral index $-p / 2 \sim-1.5$ in Eq. (22) is inconsistent with the observations $(\sim 1)$. Thus, this model should be modified, e.g., by making microphysical parameters time-dependent. In the time-dependent microphysics model, we have the observed indices $\sim-0.5$ (X-ray) and $\sim-0.7$ (optical) for $p \sim 2, \beta_{\mathrm{e}} \sim 0.5$, and $\beta_{B} \sim-0.6$ from Eqs. (30)-(32). (We adopt $p \sim 2$ to fit the post-shallow phase. See below.) Thus, the magnetic field energy also decreases during the shallow phase. It is interesting to note that the particle simulations also suggest a long term decay of magnetic fields (Silva et al. 2003; Kato 2005).

The multi-wavelength observations of the early afterglows also suggest that the shallow X-ray phase ends without any optical break (Panaitescu et al. 2006b). In the prior activity model, the X-ray and optical decay indices are $\sim-2$ and $\sim-2$, respectively, just after the shallow X-ray phase from Eqs. (27) and (28), and finally $\sim-1.7$ and $\sim-1.5$, respectively, from Eq. (29), if we use the same parameters, $p \sim 3$ and $\alpha \sim 3.8$, as in the shallow $\mathrm{X}$-ray phase. This is inconsistent with the observations. To explain the observations in this model, the power-law index of the electron distribution $p$ should finally change to the conventional value $p \sim 2$. In the time-dependent microphysics model, the chromatic X-ray break can be explained if the microphysical parameters stop evolutions after the shallow X-ray phase since the $\mathrm{X}$-ray and optical decay indices become $\sim-1$ and $\sim-0.8$, respectively, for $p \sim 2$ from Eq. (29). Note that the simplest form of the current models (the energy injection and the inhomogeneous jet model) predicts an optical break at the end of the shallow X-ray decay because the hydrodynamics changes.

The prediction of the prior activity model is a precursor produced by the external shock due to the prior explosion (see Fig. 1). Such a precursor may have evaded the detection since its luminosity could be low if the maximum Lorentz factor of the prior explosion $\gamma_{\max }$ is not so large. The prompt emission from the prior explosion may be also $\operatorname{dim}$ if $\gamma_{\max }$ is too low to avoid the compactness problem. The precursor emission peaks around the deceleration time $t_{\mathrm{dec}} \sim 10^{4} E_{\mathrm{p}, 52}^{1 / 3} \gamma_{\max , 1.5}^{-8 / 3} n^{-1 / 3} \mathrm{~s}$ in Eq. (1), and it is $t_{\mathrm{p}}-t_{\mathrm{dec}} \sim 10^{3}-10^{6} \mathrm{~s}$ before the main burst. The peak luminosity of the precursor is about $L_{\mathrm{p}} \sim \epsilon_{\mathrm{e}} E_{\mathrm{p}} / t_{\mathrm{dec}} \sim$ $10^{47} \epsilon_{\mathrm{e},-1} E_{\mathrm{p}, 52} t_{\mathrm{dec}, 4}^{-1} \mathrm{erg} \mathrm{s}^{-1}$.

In both the prior activity model and the time-dependent microphysics model, it is predicted that the optical emission from the reverse shock is suppressed (see Sect. 4). This may be relevant to the dim optical flashes from the reverse shock recently reported (Roming et al. 2005). A possibility that the prior activity is continuous is also interesting to study.

Acknowledgements. We thank Y. Z. Fan and C. Hededal for useful comments. This work is supported in part by Grant-in-Aid for the 21st Century COE "Center for Diversity and Universality in Physics" from the Ministry of Education, Culture, Sports, Science and Technology (MEXT) of Japan and also by Grantsin-Aid for Scientific Research of the Japanese Ministry of Education, Culture, Sports, Science, and Technology 18740147 (K.I.), 14047212 (K.I. and T.N.), 14204024 (K.I. and T.N.), 18740153 (R.Y.), and 17340075 (T.N.).

\section{References}

Barthelmy, S. D., Cannizzo, J. K., Gehrels, N., et al. 2005, ApJ, 635, L133 Beloborodov, A. M. 2000, ApJ, 539, L25 
Blandford, R. D. \& McKee, C. F. 1976, Phys. Fluids, 19, 1130

Burrows, D. N., Romano, P., Falcone, A., et al. 2005, Science, 309, 1833

Chincarini, G., et al. 2005 [arXiv: astro-ph/0506453]

Cusumano, G., Mangano, V., Angelini, L., et al. 2006, ApJ, 639, 316

Dai, Z. G., \& Lu, T. 1998, A\&A, 333, L87

Eichler, D., \& Waxman, E 2005, ApJ, 627, 861

Eichler, D., \& Granot, J. 2006, ApJ, 641, L5

Fan, Y. Z., \& Wei, D. M. 2005, MNRAS, 364, L42

Fan, Y. Z., \& Piran, T. 2006, MNRAS, 369, 197

Granot, J., \& Kumar, P. 2006, MNRAS, 366, L13

Granot, J., Konigl, A., \& Piran, T. 2006 [arXiv:astro-ph/0601056]

Hededal, C. 2005 [arXiv: astro-ph/0506559]

Hill, J. E., Morris, D. C., Sakamoto, T., et al. 2006, ApJ, 639, 303

Ioka, K. 2005, Prog. Theor. Phys., 114, 1317

Ioka, K., Kobayashi, S., \& Zhang, B. 2005, ApJ, 631, 429

Kato, T. N. 2005, Phys. Plasmas, 12, 080705

King, A., O’Brien, P. T., Goad, M. R., et al. 2005, ApJ, 630, L113

Kobayashi, S., \& Sari, R. 2001, ApJ, 551, 934

Kobayashi, S., Zhang, B., Mészáros, P., \& Burrows, D. N. 2005 [arXiv: astro-ph/0506157]

Kumar, P., \& Piran, T. 2000, ApJ, 532, 286

Lazzati, D. 2005, MNRAS, 357, 722

Lazzati, D., \& Begelman, M. C. 2005, ApJ, 641, 972

Lithwick, Y., \& Sari, R. 2001, ApJ, 555, 540

Lloyd-Ronning, N. M., \& Zhang, B. 2004, ApJ, 613, 477
Medvedev, M. V., \& Loeb, A. 1999, ApJ, 526, 697

Nousek, J. A., Kouveliotou, C., Grupe, D., et al. 2006, ApJ, 642, 389

Panaitescu, A., Mészáros, P., Gehrels, N., Burrows, D., \& Nousek, J. A. 2006, MNRAS, 366, 1357

Panaitescu, A., Mészáros, P., Burrows, D., et al. 2006 [arXiv: astro-ph/0604105]

Papathanassiou, H., \& Mészáros, P. 1996, ApJ, 471, L91

Perna, R., Armitage, P. J., \& Zhang, B. 2006, ApJ, 636, 29

Piro, L., De Pasquale, M., Soffitta, P., et al. 2005, ApJ, 623, 314

Rees, M. J., \& Mészáros, P. 1998, ApJ, 496, L1

Rees, M. J., \& Mészáros, P. 2000, ApJ, 545, L73

Roming, P. W. A., et al. 2005 [arXiv: astro-ph/0509273]

Sari, R. 1995, ApJ, 455, L143

Sari, R. 1997, ApJ, 489, L37

Sari, R., \& Mészáros, P. 2000, ApJ, 535, L33

Sari, R., Piran, T., \& Narayan, R. 1998, ApJ, 497, L17

Silva, L. O., Fonseca, R. A., Tonge, J. W., et al. 2003, ApJ, 596, L121

Tagliaferri, G., Goad, M., Chincarini, G., et al. 2005, Nature, 436, 985

Toma, K., Ioka, K., Yamazaki, R., \& Nakamura, T. 2006, ApJ, 640, L139

Vaughan, S., Goad, M. R., Beardmore, A. P., et al. 2006, ApJ, 638, 920

Vietri, M., \& Stella, L. 1998, ApJ, 507, L45

Yamazaki, R., Toma, K., Ioka, K., \& Nakamura, T. 2006, MNRAS, 369, 311

Yost, S. A., Harrison, F. A., Sari. R., \& Frail, D. A. 2003, ApJ, 597, 459

Zhang, B., \& Mészáros, P. 2002, ApJ, 566, 712

Zhang, B., Fan, Y. Z., Dyks, J., et al. 2006, ApJ, 642, 354 